Article

\title{
Potential Application of Gold Nanospheres as a Surface Plasmon Resonance Based Sensor for In-Situ Detection of Residual Fungicides
}

\author{
Hang Nguyen Thi Nhat ${ }^{1}$, Ngoc Thuy Trang Le ${ }^{2} \mathbb{}$, Nguyen Thi Phuong Phong ${ }^{3}$, \\ Dai Hai Nguyen ${ }^{4,5}$ and Minh-Tri Nguyen-Le ${ }^{6,7, *(D)}$ \\ 1 Faculty of Natural Sciences, Thu Dau Mot University, Thu Dau Mot City 820000, Binh Duong Province, \\ Vietnam; hangntn@tdmu.edu.vn \\ 2 Institute of Research and Development, Duy Tan University, Danang 550000, Vietnam; \\ lenthuytrang4@duytan.edu.vn \\ 3 Faculty of Chemistry, University of Science, Vietnam National University Ho Chi Minh City, \\ 227 Nguyen Van Cu Street, District 5, Ho Chi Minh City 70000, Vietnam; ntpphong@hcmus.edu.vn \\ 4 Institute of Applied Materials Science, Vietnam Academy of Science and Technology, 01 TL29, District 12, \\ Ho Chi Minh City 700000, Vietnam; nguyendaihai0511@gmail.com \\ 5 Graduate University of Science and Technology, Vietnam Academy of Science and Technology, \\ 18 Hoang Quoc Viet, Cau Giay, Hanoi 100000, Vietnam \\ 6 Laboratory of Advanced Materials Chemistry, Advanced Institute of Materials Science, \\ Ton Duc Thang University, Ho Chi Minh City 758307, Vietnam \\ 7 Faculty of Applied Sciences, Ton Duc Thang University, Ho Chi Minh City 758307, Vietnam \\ * Correspondence: nguyenleminhtri@tdtu.edu.vn
}

Received: 29 February 2020; Accepted: 8 April 2020; Published: 15 April 2020

check for updates

\begin{abstract}
It is essential to develop a simple and sensitive method to rapidly detect residual fungicides in agricultural products to protect human health. So far, little studies have been reported on potential application of gold nanospheres (AuNSps) as a surface plasmon resonance based sensor for in-situ detection of residual fungicides. Therefore, in this study, we investigated the potential application of AuNSps as a surface plasmon resonance based sensor for in-situ detection of fungicides. AuNSps were successfully synthesized via a seed-mediated method with some modifications. Firstly, gold nanoseeds were made during the reduction of chloroauric acid by trisodium citrate dihydrate (TSC). Then, AuNSps were grown from the seeds by using $\mathrm{HAuCl}_{4}$, TSC and EDTA. AuNSps were subsequently dropped on a glass substrate before covered by thiophanate methyl, a broad-spectrum systemic fungicide. The AuNSps coated glass substrate was subsequently dried in the air for further surface-enhanced Raman spectroscopy (SERS) measurements. Optical properties, shape and size of AuNSps were confirmed by UV-vis spectroscopy, XRD, SEM-EDX and TEM. The results showed that AuNSps were successfully synthesized with the size of $53 \mathrm{~nm}$, and their resonance peak was located at $560 \mathrm{~nm}$. The Raman signal intensity of thiophanate methyl covered on AuNSps is higher than that without AuNSps, indicating SERS effects of AuNSps deposited glass substrate.
\end{abstract}

Keywords: gold; nanosphere; SERS; fungicides; Raman

\section{Introduction}

The development of science and technologies has led to the introduction of modern analytical methods with high sensitivity and accuracy, such as high performance liquid chromatography (HPLC), fluorescence assay (FL), high performance liquid chromatography mass spectrometry/mass spectrometry (HPLC-MS/MS), electrochemical assay (EC) and gas chromatography/mass spectrometry 
(GC/MS). However, these methods have some limitations such as complicated sample preparation and analysis processes that require operators to be highly qualified, time-consuming operation, high consumption of organic solvents and restricted on-site measurement. Since the discovery of Raman spectroscopy took placed in early 1928, Raman spectroscopy has been thought to be a promising method to overcome these disadvantages [1]. However, the application of Raman spectroscopy in analytical field was not popular at that time due to its low sensitivity as a result of low intensity of Raman scattered light as compared with the incident laser. In order to increase the intensity of Raman scattered light, a high-intensity laser should be used [2]. However, the sensitivity of the method is not really high until the study of surface-enhanced Raman spectroscopy (SERS) on the electrode silver anode of DL Jeanmaire was published in 1977 [3]. SERS is thought to be the result of the enhancement of Raman scattered light due to both physical and chemical factors [4]. The physical factor is the enhancement of the electromagnetic field on the nanoparticle surface due to the surface plasmon resonance, while the chemical factor is the electrical resonance and charge transfer between the adsorbent molecules and the metal surface [5]. SERS can be observed on the surface of several of nanoparticles such as Au, $\mathrm{Ag}, \mathrm{Pt}, \mathrm{Cu}$ and $\mathrm{Pd}$ [6-11]. Among them, gold nanoparticles with non-toxicity and high bioactivity have gained much interest, and they present a wide range of applications in modern medical and biological studies such as drug, DNA and antigens delivery; biosensorics, genomics, immunoanalysis, detection of microorganisms and cancer cells and bioimaging [12]. It has been argued that the shape, size and structure of gold nanoparticles determine their chemical and physical properties $[13,14]$. Therefore, shape-, size- and structure-controlled synthesis of gold nanoparticles is urgently important and considerably challenging. To date, possible wet synthesis methods have been used to fabricate gold nanoparticles of various controlled sizes, shapes and structures such as nanospheres, nanorods, nanoshells, nanocages, nanostars, etc. [15].

Thiophanate methyl whose chemical structure is shown in Figure 1 is known as a member of the benzimidazole group of fungicides [16]. It is a broad-spectrum systemic fungicide that has been employed to control the pathogens recently. However according to the office of Environmental Health Hazard Assessment (OEHHA), long exposure to residue from thiophanate methyl in agricultural products may cause serious effects on human health [16]. Therefore, it is essential to develop a sensitive on-site method to detect residual thiophanate methyl in agricultural products to prevent humans from being exposed to thiophanate methyl [17]. The analysis of thiophanate methyl residues by $\mathrm{Au}$ nanospheres (AuNSps) - supported Raman spectroscopy_has the potential to be an accurate portable analytical method. So far, little studies have been reported on the potential application of AuNSps as a surface plasmon resonance based sensor for in-situ detection of residual fungicides.

In this work, AuNSps were synthesized by a facile method using TSC as a reducing agent and EDTA as a protective agent during the nucleation stage. The as-synthesized AuNSps were used as a platform to investigate the SERS signal in thiophanate methyl fungicide analysis.

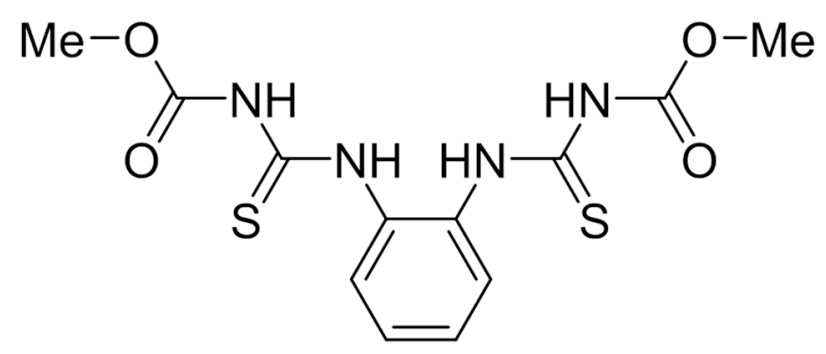

Figure 1. The chemical structure of thiophanate methyl. 


\section{Materials and Methods}

\subsection{Chemicals and Characterization}

Tetrachloroauric (III) acid trihydrate $\left(\mathrm{HAuCl}_{4} \cdot 3 \mathrm{H}_{2} \mathrm{O}, \geq 99.5 \%\right)$ and ethylenediaminetetraacetic acid (EDTA, $\geq 98.0 \%$ ) were purchased from Merck (Darmstadt, Germany). Trisodium citrate dihydrate (TSC, $\geq 99.0 \%$ ) were purchased from Prolabo (Kennersburg, NJ, USA). Deionized (DI) water (>18 MW, Millipore, Darmstadt, Germany) was used during the synthesis of AuNSps. A commercial product, called TOPSIN M 70WP (containing 70\% of thiophanate methyl), was purchased from Nippon Soda Company, Ltd. All reagents were used without further purification.

The morphology of the as-synthesized samples was obtained by scanning electron microscopy (Hitachi, Horiba S-4300) coupling with energy-dispersive X-ray (EDX) spectroscopy operated at $20 \mathrm{kV}$ of the incident electron beam energy. Transmission electron microscope (TEM) images were obtained with a JEOL JEM-1400 (120 kV). X-ray diffractometer (XRD, Bruker D8 advance powder diffractometer model) with $\mathrm{Cu} \mathrm{K} \alpha$ radiation $(\lambda=1.54056 \AA$ ) operated at $40 \mathrm{kV}$ and $30 \mathrm{~mA}$ with a scanning rate of $0.02^{\circ}$ per step in the $2 \theta$ range of $10^{\circ} \leq 2 \theta \leq 90^{\circ}$ was carried out to characterize the crystal structure of AuNSps. UV-vis spectroscopy (UV-Vis-NIR-V670, JASCO, Tokyo, Japan) was utilized to investigate the optical properties of AuNSps. The Raman spectra of AuNSps samples were recorded on a LabRam HR micro-Raman instrument with a $532 \mathrm{~nm}$ Ar+ ion laser at room temperature.

\subsection{Synthesis of Au Nanoseeds and AuNSps}

Au nanoseeds were synthesized by the Turkevich method [15] with some modifications. Firstly, $100 \mu \mathrm{L}$ of $25 \mathrm{mM} \mathrm{HAuCl}_{4}$ was mixed with $10 \mathrm{~mL}$ of DI water, and the obtained solution was boiled at $100{ }^{\circ} \mathrm{C}$. Under vigorous stirring $300 \mu \mathrm{L}$ of $1 \%$ TSC was then added to the solution. The color of the solution will change from colorless to reddish. After cooling at room temperature, the solution was kept in dark for 30 min for seeding crystallization of Au nanoseeds.

For the synthesis of AuNSps, $50 \mu \mathrm{L}$ of the Au nanoseed solution and $100 \mu \mathrm{L}$ of $25 \mathrm{mM} \mathrm{HAuCl}_{4}$ were firstly added into $100 \mathrm{~mL}$ of DI water under vigorous stirring. Next, $20 \mu \mathrm{L}$ of $1 \%$ TSC and $100 \mu \mathrm{L}$ of 0.1 M EDTA were added to the mixture. The solution was kept in the dark for 30 min for stabilization. The whole procedure of AuNSps synthesis was illustrated in Figure 2.

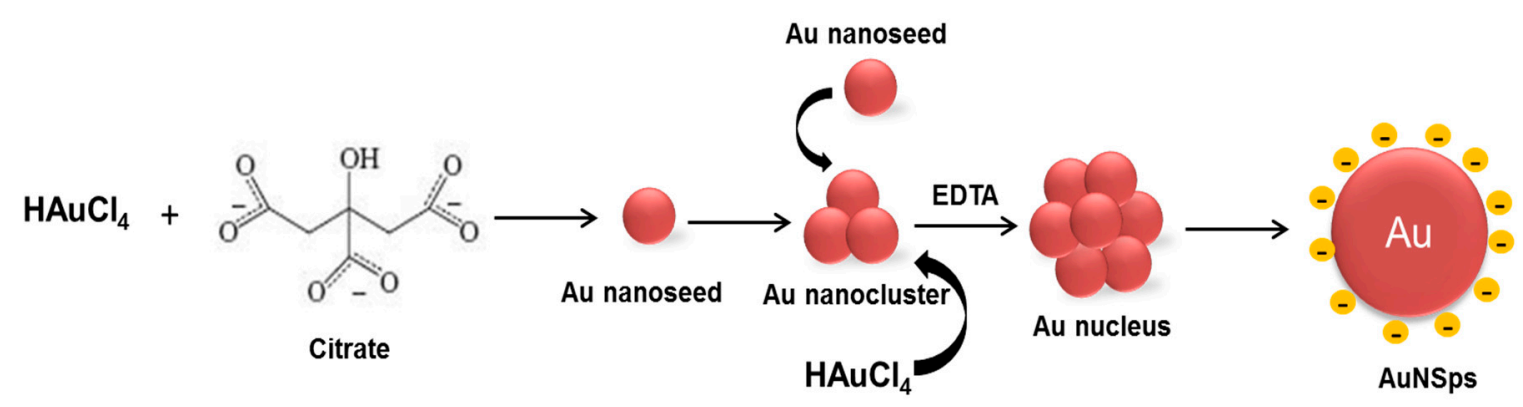

Figure 2. Fabrication process of gold nanospheres (AuNSps).

\subsection{SERS Measurement}

The Raman spectra of thiophanate methyl were recorded on an AuNSps coated glass substrate in order to study the SERS effects. To prepare AuNSps coated glass substrate, the substrate was firstly rinsed with a 3:1 (v/v) mixture of concentrated $\mathrm{H}_{2} \mathrm{SO}_{4}$ and $30 \% \mathrm{H}_{2} \mathrm{O}_{2}$ in $15 \mathrm{~min}$, subsequently washed with DI water and dried in the air. Twenty microliters of AuNSps solution was gently dropped onto the clean substrate homogeneously, and the substrate was then dried in the air. Next, Twenty microliters of thiophanate methyl (in DI water) was gently dropped onto the AuNSps coated glass substrate, and the substrate was subsequently dried in the air for further SERS measurements. For comparison, Raman spectra of a clean substrate, AuNSps and thiophanate methyl solutions $\left(10^{-4} \mathrm{M}\right.$ and $\left.0.1 \mathrm{M}\right)$ were also recorded. The procedure of SERS measurement was illustrated in Scheme 1. 


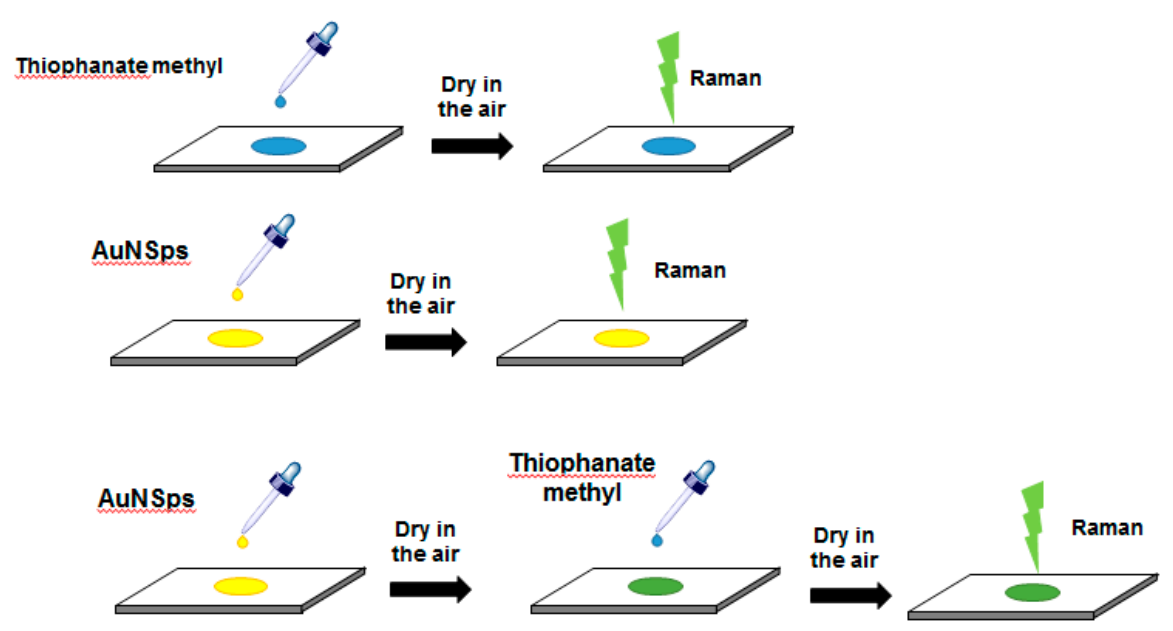

Scheme 1. Surface-enhanced Raman spectroscopy (SERS) measurement procedure.

\section{Results and Discussion}

\subsection{Synthesis of AuNSps}

Optical properties of AuNSps and Au nanoseeds were studied through UV-Vis spectra. As shown in Figure 3, the spectra of Au nanoseeds exhibited a characteristic peak at $521 \mathrm{~nm}$, while the resonance peak of AuNSps located at $560 \mathrm{~nm}$ [18]. The red-shift of surface plasmon resonance peak indicated the crystal growth of Au nanoparticles from Au nanoseeds. It is widely accepted that the optical properties of AuNSps are highly dependent on the nanoparticle diameter [19]. Smaller nanospheres primarily absorb light and have absorption peaks near $520 \mathrm{~nm}$, while larger spheres exhibit light scattering and have significantly broaden and red-shifted absorption peaks. Larger spheres scatter more light both because they have larger optical cross sections, and also because the ratio of scattering to total extinction increases with size $[20,21]$.

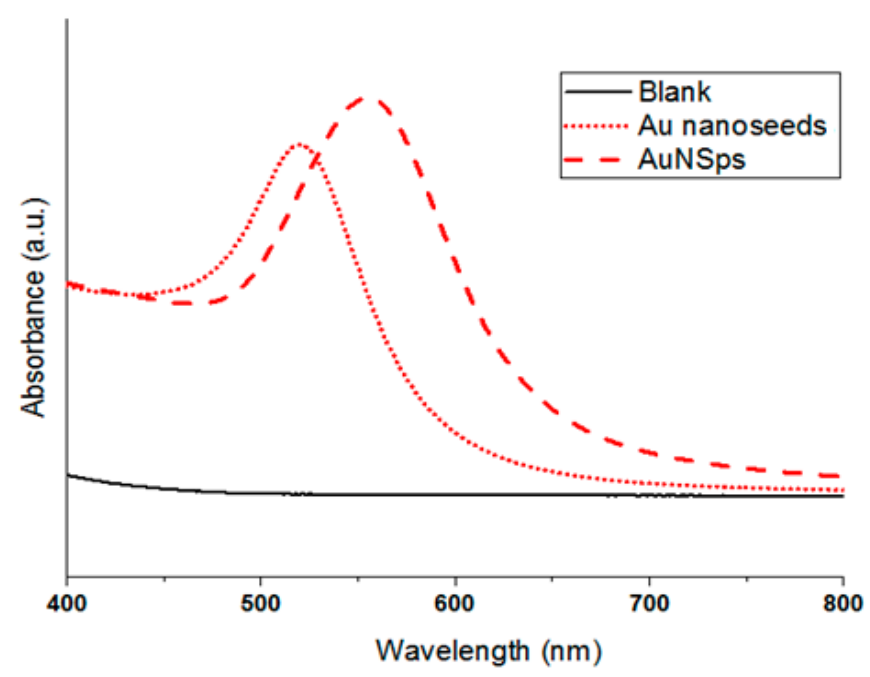

Figure 3. UV-Vis spectra of Au nanoseeds and AuNSps.

The solution of Au nanoseeds consisted of clusters of Au nanosized particles with the average diameter of $18.27 \pm 0.08 \mathrm{~nm}$ (Figure $4 \mathrm{a}, \mathrm{b}$ ). Nanoseeds with the crystalline lattices of $0.231 \mathrm{~nm}$, corresponding to the (111) crystal plane of $\mathrm{Au}$, is clearly observed in the HRTEM image (Figure 4a, the inset) $[22,23]$. The TEM images of AuNSps revealed monodisperse spherical nanostructures with the average diameter of $53.52 \pm 0.36 \mathrm{~nm}$ (Figure $4 \mathrm{c}, \mathrm{d}$ ). 

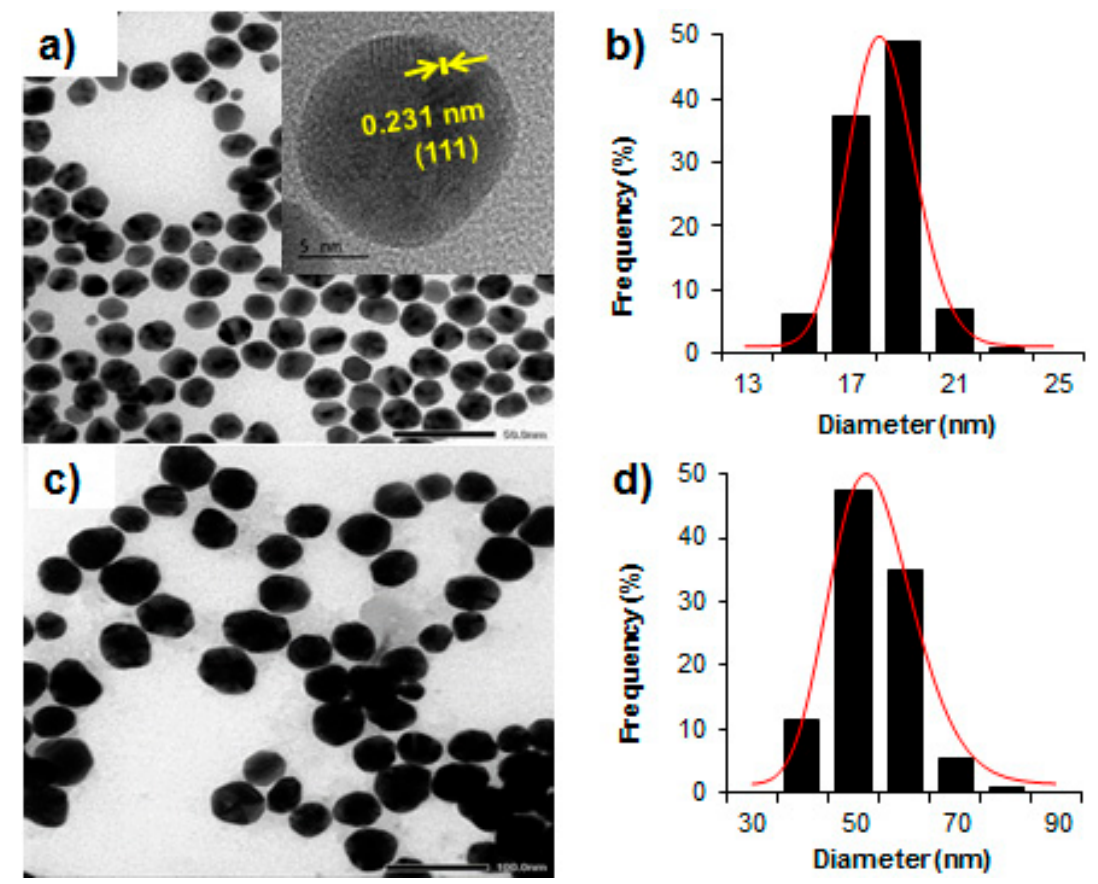

Figure 4. TEM image and particle size distribution of $(\mathbf{a}, \mathbf{b})$ Au nanoseeds, and (c,d) AuNSps. The inset: HRTEM image of Au nanoseeds.

Figure 5a revealed XRD pattern of AuNSps with characteristic peaks of Au at $2 \theta=38.1^{\circ}(111)$, $44.3^{\circ}$ (200), $64.5^{\circ}$ (220) and $77.7^{\circ}$ (311) [24]. No impurities were found in XRD spectra of AuNSp. The EDX spectrum in Figure $5 \mathrm{~b}$ represented that $\mathrm{Au}$ and $\mathrm{Si}$ were the major elements of AuNSps coated glass substrate, indicating successful coating of the glass substrate with AuNSps.

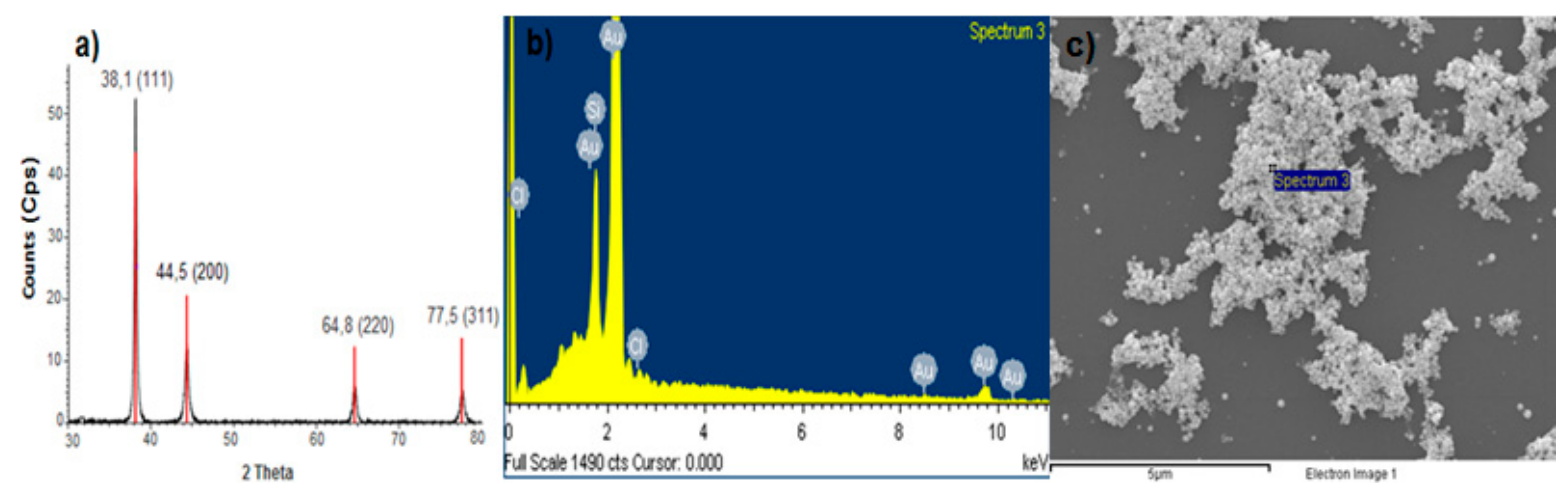

Figure 5. (a) XRD spectra of AuNSps, (b) EDX spectra and (c) SEM image of AuNSps coated glass.

\subsection{SERS Measurement}

The Raman spectra of $0.1 \mathrm{M}$ thiophanate methyl in Figure 6a showed the signals at $613 \mathrm{~cm}^{-1}$, $726 \mathrm{~cm}^{-1}, 779 \mathrm{~cm}^{-1}, 898 \mathrm{~cm}^{-1}, 959 \mathrm{~cm}^{-1}$ and $1538 \mathrm{~cm}^{-1}$, which are ascribed to $-\mathrm{N}-\mathrm{C}=\mathrm{S}$ deformation, $\mathrm{N}-\mathrm{H}$ wagging of $-(\mathrm{C}=\mathrm{O})-\mathrm{NH}-(\mathrm{C}=\mathrm{S})-, \mathrm{C}=\mathrm{S}$ stretching, $\mathrm{C}-\mathrm{S}$ stretching, $\mathrm{C}=\mathrm{S}$ and $\mathrm{C}-\mathrm{N}$, respectively. Moreover, other signals of $=\mathrm{C}-\mathrm{H}$ deformation, $\mathrm{N}-\mathrm{C}-\mathrm{N}$ asymmetric stretching, $\mathrm{C}-\mathrm{O}, \mathrm{C}-\mathrm{O}-\mathrm{C}, \mathrm{C}=\mathrm{C}, \mathrm{C}=\mathrm{O}$ and N-H were also observed at $1039 \mathrm{~cm}^{-1}, 1154 \mathrm{~cm}^{-1}, 1267 \mathrm{~cm}^{-1}, 1298 \mathrm{~cm}^{-1}, 1601 \mathrm{~cm}^{-1}$ and 1708 $\mathrm{cm}^{-1}$, respectively [25]. As shown in Figure 6d,e, the glass substrate or AuNSps itself did not give any Raman signals of thiophanate methyl indicating thiophanate methyl-free surfaces. It should be noted that even though in the presence of thiophanate methyl, those signals could not be observed when the concentration of thiophanate methyl was as low as $10^{-4} \mathrm{M}$ (Figure 6c), probably due to being under the detection limit. Interestingly, at the same concentration of thiophanate methyl, most of 
the characteristic Raman peaks of thiophanate methyl were clearly observed after the deposition of thiophanate methyl onto the AuNSps coated glass substrate (Figure 6b), indicating the SERS effect of AuNSps. Therefore, this effect is useful for the detection at a low level of thiophanate methyl, particularly under the detection limit of thiophanate methyl.

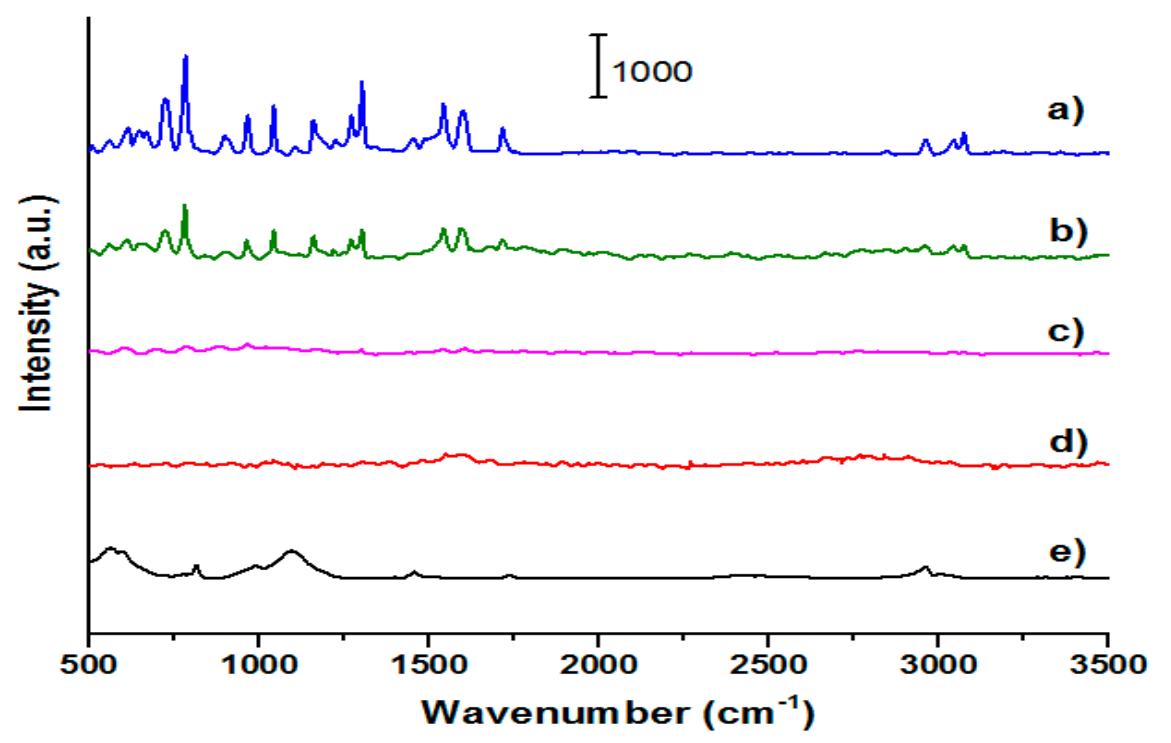

Figure 6. Raman spectra of (a) $0.1 \mathrm{M}$ thiophanate methyl, (b) $10^{-4} \mathrm{M}$ thiophanate methyl deposited onto AuNSps coated glass substrate, (c) $10^{-4} \mathrm{M}$ thiophanate methyl, (d) AuNSps, and (e) glass substrate.

To elucidate the sensitivity of the method, $20 \mu \mathrm{L}$ of thiophanate methyl solutions with concentrations ranging from 11 to $30 \mu \mathrm{M}$ were deposited onto AuNSps coated glass substrates, which serve as Raman probes. The corresponding Raman spectra of thiophanate methyl were collected in Figure 7. It can be seen that the characteristic peaks of thiophanate methyl could be clearly identified in all Raman spectra. Typically, the intensity of SERS signals increased with the increasing investigated concentrations of thiophanate methyl. The limit of detection (LOD) was determined as low as $17 \mu \mathrm{M}$ at which the signal to noise $(\mathrm{S} / \mathrm{N})$ ratio for the peak $779 \mathrm{~cm}^{-1}$ dropped to 3 . The limit of quantification (LOQ), therefore, was estimated as $57 \mu \mathrm{M}$.

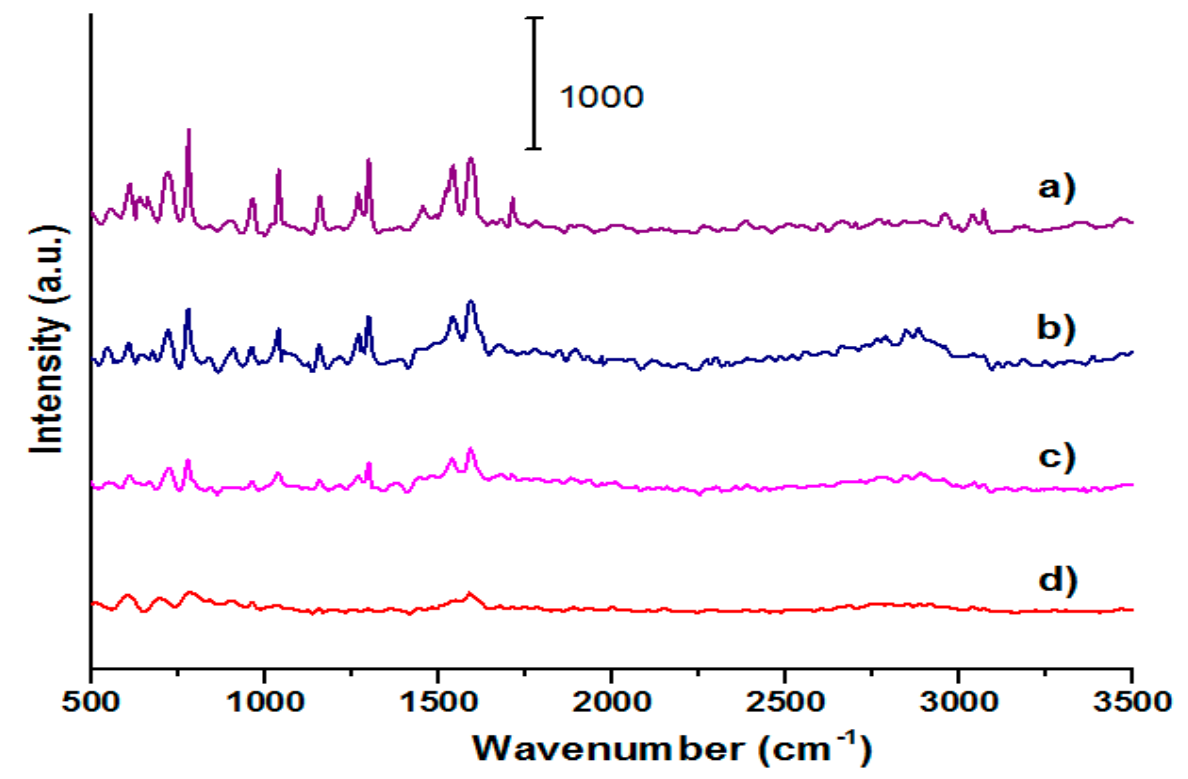

Figure 7. Raman spectra of thiophanate methyl collected on AuNSps coated glass substrate at different concentrations of thiophanate methyl: (a) $30 \mu \mathrm{M}$, (b) $23 \mu \mathrm{M}$, (c) $17 \mu \mathrm{M}$ and (d) $11 \mu \mathrm{M}$. 
The reproducibility of the measurement was also evaluated by collecting SERS spectra of $30 \mu \mathrm{M}$ thiophanate methyl at 10 random locations on the as-fabricated substrate, as shown in Figure 8 . The relative standard deviation (RSD) was found to be $4.8 \%$, which indicates a good reproducibility for routine SERS analysis.

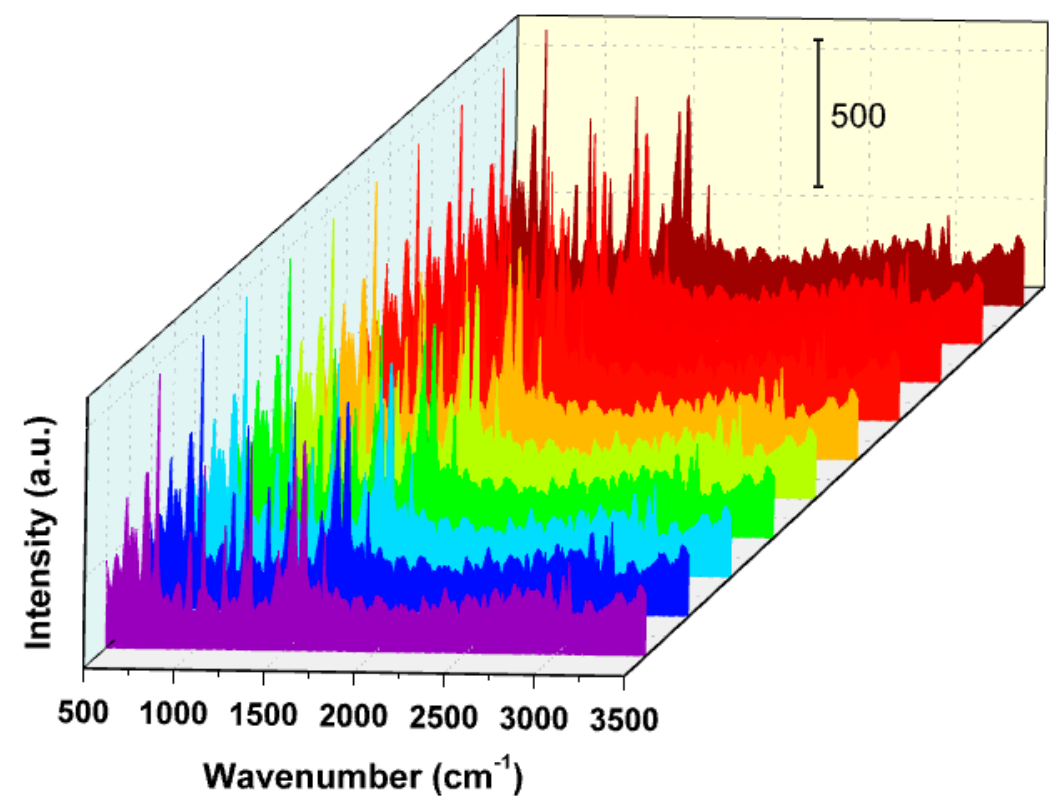

Figure 8. Raman spectra of $30 \mu \mathrm{M}$ thiophanate methyl collected at 10 random locations on AuNSps coated glass substrate.

\section{Conclusions}

AuNSps were successfully synthesized from crystallization of Au nanoseeds through a facile seed-mediated method using TSC as a primary reducing agent and EDTA as a protective agent during the nucleation stage. The as-synthesized AuNSps showed SERS effect for thiophanate methyl with low LOD of thiophanate methyl down to $17 \mu \mathrm{M}$. The Raman signal intensity of thiophanate methyl covered on AuNSps was higher than that without AuNSps, which revealed the potential application of AuNSps as a surface plasmon resonance based sensor for in-situ detection of fungicides with high sensitivity and reproducibility.

Author Contributions: Conceptualization, N.T.P.P. and D.H.N.; Formal analysis, H.N.T.N. and M.-T.N.-L.; Funding acquisition, D.H.N.; Investigation, H.N.T.N. and T.T.L.N.; Methodology, N.T.P.P. and D.H.N.; Project administration, D.H.N.; Supervision, M.-T.N.-L.; Writing—original draft, H.N.T.N.; Writing—review and editing, M.-T.N.-L. All authors have read and agreed to the published version of the manuscript.

Funding: This project is funded by the Thu Dau Mot University.

Acknowledgments: The authors would like to thank Cuu Khoa Nguyen and Ngoc Quyen Tran-Board of Directors of Institute of Applied Materials Science, Vietnam Academy of Science and Technology, Ho Chi Minh City—-for their helpful advice and technical support for this study.

Conflicts of Interest: The authors declare no conflict of interest.

\section{References}

1. Panneerselvam, R.; Liu, G.K.; Wang, Y.H.; Liu, J.Y.; Ding, S.Y.; Li, J.F.; Wu, D.Y.; Tian, Z.Q. Surface-enhanced Raman spectroscopy: Bottlenecks and future directions. Chem. Commun. 2018, 54, 10-25. [CrossRef] [PubMed]

2. Ye, K.; Li, K.; Lu, Y.; Guo, Z.; Ni, N.; Liu, H.; Huang, Y.; Ji, H.; Wang, P. An overview of advanced methods for the characterization of oxygen vacancies in materials. Trac Trends Anal. Chem. 2019, 116, 102-108. [CrossRef] 
3. Sharma, B.; Frontiera, R.R.; Henry, A.-I.; Ringe, E.; van Duyne, R.P. SERS: Materials, applications, and the future. Mater. Today 2012, 15, 16-25. [CrossRef]

4. Le Ru, E.C.; Blackie, E.; Meyer, M.; Etchegoin, P.G. Surface Enhanced Raman Scattering Enhancement Factors: A Comprehensive Study. J. Phys. Chem. C 2007, 111, 13794-13803. [CrossRef]

5. Moskovits, M. Surface-enhanced Raman spectroscopy: A brief perspective. In Surface-Enhanced Raman Scattering; Springer: Berlin/Heidelberg, Germany, 2006; pp. 1-17.

6. Pacheco-Londono, L.C.; Aparicio-Bolano, J.; Primera-Pedrozo, O.M.; Hernandez-Rivera, S.P. Growth of Ag, $\mathrm{Au}, \mathrm{Cu}$, and Pt nanostructures on surfaces by micropatterned laser-image formations. Appl. Opt. 2011, 50, 4161-4169. [CrossRef]

7. Li, X.; Zhang, S.; Yu, Z.; Yang, T. Surface-enhanced Raman spectroscopic analysis of phorate and fenthion pesticide in apple skin using silver nanoparticles. Appl. Spectrosc. 2014, 68, 483-487. [CrossRef]

8. Thi, H.N.; Nguyen, D.H.; Vu, M.T.; Tran, H.N.; Tran, L.P.; Nguyen-Thi, N.T.; Le Ngoc, T.T.; Nguyen-Le, M.T. Fabrication Process and Characterization of AgNPs/PVA/Cellulose as a SERS Platform for In-situ Detection of Residual Pesticides in Fruit. Mater. Res. Express 2020. [CrossRef]

9. Tri, N.L.M.; Jitae, K.; van Thuan, D.; Huong, P.T.; al Tahtamouni, T.M. Improved photocatalytic decomposition of methyl ethyl ketone gas from indoor air environment by using $\mathrm{TiO}_{2} /$ graphene oxide. Mater. Res. Express 2019, 6, 105509.

10. Anh, M.N.T.; Nguyen, D.T.D.; Thanh, N.V.K.; Phong, N.T.P.; Nguyen, D.H.; Nguyen-Le, M.-T. Photochemical Synthesis of Silver Nanodecahedrons under Blue LED Irradiation and Their SERS Activity. Processes 2020, 8 , 292.

11. Tri, N.L.; Kim, J.; Giang, B.L.; Al Tahtamouni, T.M.; Huong, P.T.; Lee, C.; Viet, N.M. Ag-doped graphitic carbon nitride photocatalyst with remarkably enhanced photocatalytic activity towards antibiotic in hospital wastewater under solar light. J. Ind. Eng. Chem. 2019, 80, 597-605.

12. Farka, Z.; Juř́k, T.; Kovář, D.; Trnková, L.; Skládal, P. Nanoparticle-Based Immunochemical Biosensors and Assays: Recent Advances and Challenges. Chem. Rev. 2017, 117, 9973-10042. [CrossRef] [PubMed]

13. Luty-Błocho, M.; Fitzner, K.; Hessel, V.; Löb, P.; Maskos, M.; Metzke, D.; Pacławski, K.; Wojnicki, M. Synthesis of gold nanoparticles in an interdigital micromixer using ascorbic acid and sodium borohydride as reducers. Chem. Eng. J. 2011, 171, 279-290. [CrossRef]

14. Dozol, H.; Mériguet, G.; Ancian, B.; Cabuil, V.; Xu, H.; Wang, D.; Abou-Hassan, A. On the synthesis of Au nanoparticles using EDTA as a reducing agent. J. Phys. Chem. C 2013, 117, 20958-20966. [CrossRef]

15. Turkevich, J.; Stevenson, P.C.; Hillier, J. A study of the nucleation and growth processes in the synthesis of colloidal gold. Discuss. Faraday Soc. 1951, 11, 55-75. [CrossRef]

16. Chayata, H.; Lassalle, Y.; Nicol, É.; Manolikakes, S.; Souissi, Y.; Bourcier, S.; Gosmini, C.; Bouchonnet, S. Characterization of the ultraviolet-visible photoproducts of thiophanate-methyl using high performance liquid chromatography coupled with high resolution tandem mass spectrometry-Detection in grapes and tomatoes. J. Chromatogr. A 2016, 1441, 75-82. [CrossRef]

17. OEHHA. Proposition 65 Maximum Allowable Dose Level (MADL) for Reproductive Toxicity for Thiophanate-Methyl for the Oral Route of Exposure; OEHHA: Sacramento, CA, USA, 2004.

18. Meng, F.; Ma, X.; Duan, N.; Wu, S.; Xia, Y.; Wang, Z.; Xu, B. Ultrasensitive SERS aptasensor for the detection of oxytetracycline based on a gold-enhanced nano-assembly. Talanta 2017, 165, 412-418. [CrossRef]

19. Sharifi, M.; Hosseinali, S.H.; Alizadeh, R.H.; Hasan, A.; Attar, F.; Salihi, A.; Shekha, M.S.; Amen, K.M.; Aziz, F.M.; Saboury, A.A.; et al. Plasmonic and chiroplasmonic nanobiosensors based on gold nanoparticles. Talanta 2020, 212, 120782. [CrossRef]

20. Shafiqa, A.R.; Aziz, A.A.; Mehrdel, B. Nanoparticle Optical Properties: Size Dependence of a Single Gold Spherical Nanoparticle. J. Phys. Conf. Ser. 2018, 1083, 012040. [CrossRef]

21. Gold Nanoparticles: Optical Properties. Available online: https://nanocomposix.eu/pages/gold-nanoparticlesoptical-properties (accessed on 5 April 2020).

22. Chang, C.-C.; Wu, H.-L.; Kuo, C.-H.; Huang, M.H. Hydrothermal Synthesis of Monodispersed Octahedral Gold Nanocrystals with Five Different Size Ranges and Their Self-Assembled Structures. Chem. Mater. 2008, 20, 7570-7574. [CrossRef]

23. Esparza, R.; Rosas, G.; Fuentes, M.L.; Ramírez, J.S.; Pal, U.; Ascencio, J.A.; Pérez, R. Synthesis of gold nanoparticles with different atomistic structural characteristics. Mater. Charact. 2007, 58, 694-700. [CrossRef] 
24. Ogundare, O.D.; Akinribide, O.J.; Adetunji, A.R.; Adeoye, M.O.; Olubambi, P.A. Crystallite size determination of thermally deposited Gold Nanoparticles. Procedia Manuf. 2019, 30, 173-179. [CrossRef]

25. Socrates, G. Infrared and Raman Characteristic Group Frequencies: Tables and Charts; John Wiley \& Sons: Chichester, UK, 2004. 